\title{
Electrocardiography may be a useful tool in the diagnosis of early mild canine heartworm disease
}

\author{
Edward M. Onyango
}

\begin{abstract}
Department of Health Sciences, East Tennessee State University, Johnson City, TN, USA, Faculty of Veterinary Medicine, University of Nairobi, Kenya
\end{abstract}

Received November 11, 2009

Accepted September 21, 2010

\begin{abstract}
This study was carried out to determine the usefulness of standard electrocardiography for the diagnosis of early canine heartworm disease. Baseline electrocardiograms were recorded in 12 dogs. Thirty artificial Dirofilaria immitis worms were inserted in the pulmonary artery of each dog. New electrocardiograms were recorded on days 1-4, 7, 14, 21, 28 and 35 after insertion of worms. A significant attenuation of amplitude of Q wave in lead I was recorded on days 1-4, 7, 14, 21, 28 and 35; of R wave in lead II on days 21, 28 and 35; and of S wave in lead aVR on days 7, 14, 21, 28 and 35. Duration of the QRS complex in lead AVF was significantly prolonged on day 14. In $41.6 \%$ of the dogs, right axis deviation was recorded. These results suggest that for a dog whose normal baseline standard electrocardiogram is known, a comparison of the QRS complex of the baseline with those of subsequent standard electrocardiograms may suggest early canine heartworm disease when there is an attenuation of amplitude of Q wave in lead I, R wave in lead II and $\mathrm{S}$ wave in lead aVR. An additional indication can be right axis deviation.
\end{abstract}

Canine heartworm, dirofilaria, dirofilariasis, ECG changes, dog

Canine heartworm disease (CHD) is an important veterinary disease. It has been reported to occur in most coastal areas of the world (Otto and Jackson 1975; Knight 1983). Dirofilaria worms are the cause of CHD and the worms are spread by several species of mosquitoes. It has been suggested that the average number of adult Dirofilaria immitis worms to be found in mild cases of CHD is 25-30 (Otto and Jackson 1975). The clinical signs in early CHD are not very helpful for diagnosis since they are not specific and the pathology of the disease is usually well advanced before the disease is clinically manifested (Otto and Jackson 1975). Radiographic examination is useful only when the worms have caused some pathologic changes in the heart or pulmonary artery. Examination for microfilariae using for example the modified Knott's method is useful if worms are mature and producing microfilariae. Angiography and ultrasonography (echocardiography) can be useful but it is expensive. Serologic methods detecting specific antigens from adult female heartworms (using for example commercial antigen detection kits; Atkins 2003) is not very useful in early cases of CHD. Molecular biology techniques using PCR have also been used for diagnosis of dirofilariasis (Rishniw et al. 2006). Because the diagnosis of early CHD is sometimes missed (Strickland 2008), there is need to test a variety of methods that can be used as early pointers to a diagnosis of early mild CHD.

Electrocardiography has been used for diagnosis of CHD. However, it is usually useful when the worms have caused some pathology in the heart and pulmonary artery. There has been no report on the use of electrocardiography as an aid in the diagnosis of CHD during the early stages of the progression of the disease. The aim of this study is to use a standard electrocardiogram, by using a single channel electrocardiograph, for the diagnosis of early CHD. In this study such electrocardiograph was used because most veterinary offices, especially in developing countries and in rural areas, will most likely own one and will be able to do, at least, a basic electrocardiogram on the dogs they see. Radiographic polyvinyl chloride threads have been used as artificial Dirofilaria immitis worms (AW) to simulate

Address for correspondence:

Edward M. Onyango

Department of Health Sciences

East Tennessee State University

Box 70673, Johnson City, TN 37614, USA

Fax: 1-423-439-4562

E-mail: onyango@etsu.edu

http://www.vfu.cz/acta-vet/actavet.htm 
the effects of the presence of worms within the pulmonary artery (Atwell et al. 1985). In this study, $30 \mathrm{AW}$ were used to induce mild CHD.

\section{Materials and Methods}

Twelve adult mixed-breed dogs ( 7 males, 5 females) from a local dog shelter in Nairobi, Kenya were used in this study. The dogs were examined to determine their health status at the Small Animal Clinic in the Faculty of Veterinary Medicine at the University of Nairobi. Only dogs that were healthy were used for this study. Their ages (using their dentition) ranged from 1-3 years and their average body weight was $17.1 \mathrm{~kg}$. The dogs were washed, dewormed and vaccinated against canine distemper, adenovirus infections, leptospirosis, and rabies. They were allowed a one week acclimation period before experimentation. The dogs were fed a balanced dog food (Besbix ${ }^{\circledR}$, Proctor and Allan Ltd., Nairobi, Kenya) supplemented with beef once a day. Water was supplied ad libitum.

A day before surgical insertion of the artificial worms into the right heart, a baseline ECG (electrocardiogram) was taken from each dog. ECGs were taken by placing and restraining each dog in right lateral recumbency with its head in the normal carriage position, its limbs at right angles to the trunk and its tail restrained together with the hind limbs. A single channel digital electrocardiograph (Kenz-ECG-103, Suzuken Co. Ltd, Nagoya, Japan) was used to record the following standard limb leads: leads I, II, III, aVR, aVL and aVF.

The dogs were fasted overnight and anaesthesia was induced and maintained with $6 \%$ pentobarbitone sodium (Saggatal ${ }^{\circledR}$, RMB Animal Health Ltd., Dagenham, England) given intravenously. Each dog was prophylactically (and also for 7 days postoperatively) treated with a combination of 400000 units of procaine penicillin $G$ and $0.5 \mathrm{~g}$ of dihydrostreptomycin sulphate (Combiotic ${ }^{\circledR}$, Pfizer, New York, U.S.A.) given intramuscularly. The right jugular vein was surgically exposed and 2 loose ligatures were placed cranially and caudally to the intended incision site on the jugular vein. An incision (about $0.5 \mathrm{~cm}$ long) was made through the jugular wall between the 2 ligatures. The cranial ligature was tightened and tied so as to close the cranial end of the vein. Heparinized Ringer's solution (Infusion Kenya Ltd., Nairobi, Kenya) was injected into the jugular vein then 30 sterile AW were inserted, using an alligator forceps, through the jugular venotomy site into the right atrium, from where the flow of blood would carry them distally to localize in the pulmonary artery. The AW insertion was done under fluoroscopic guidance using a high contrast image intensifier (IA-9W series, Shimadzu Corp., Tokyo, Japan) connected to an X-ray monitor (XT-1000 AII series, Mitsubishi Electric Corp., Tokyo, Japan). The caudal ligature on the jugular vein was tightened and the skin incision was closed. Skin sutures were removed on day 14 after the insertion procedure.

ECGs for each dog were taken post-operatively on day 1-4, 7, 14, 21, 28 and 35. At the end of the study all dogs were euthanized using an overdose of pentobarbitone sodium (Euthatal ${ }^{\circledR}$, RMB Animal Health Ltd., Dagenham, England) given intravenously. Necropsy was performed on all the dogs with special attention being given to the hearts, great vessels and lungs. All the study procedures were approved by the Deans' committee of the University of Nairobi.

The amplitude and duration of the electrocardiographic complexes were measured to the nearest $0.05 \mathrm{mV}$ and $0.01 \mathrm{~s}$, respectively, on a section of the trace with an even baseline and free of artifacts. For each measurement, at least three sets of 3 separate electrocardiographic complexes taken from 3 different locations on the ECG were examined and measured and their average value was recorded.

The mean electrical axis (MEA) in the frontal plane was determined using the inspection method (Ettinger and Suter 1970; Bolton 1975). The normal MEA was taken from $+40^{\circ}$ to $+100^{\circ}$; right axis deviation from +101 ${ }^{\circ}$ to $-90^{\circ}$ and left axis deviation from $-89^{\circ}$ to $+39^{\circ}$ (Ettinger and Suter 1970; Bolton 1975). Briefly, the QRS complex of the six leads of the Bailey's hexaxial lead system (I, II, III, aVR, aVL, and aVF) were examined to identify the isolectric or nearly isoelectric lead among them. This would be the lead whose positive and negative deflections of the QRS complex are almost equal. The MEA would run perpendicular to the isolectric lead (Bolton 1975). Using the six-axis reference chart that shows how the six leads are positioned in a circle relative to each other, the lead perpendicular to the isoelectric lead was identified. In the six-axis reference chart each lead has a positive and negative pole. The degree values of the different leads starting with lead I and going clockwise are: lead I, $0^{\circ}$ and $\pm 180^{\circ}$; lead aVR, $+30^{\circ}$ and $-150^{\circ}$; lead II, $+60^{\circ}$ and $-120^{\circ}$; lead aVF, $+90^{\circ}$ and $-90^{\circ}$; lead III, $+120^{\circ}$ and $-60^{\circ}$; lead $\mathrm{aVL},+150^{\circ}$ and $-30^{\circ}$. After determining the lead along which the MEA is running, its direction (whether to the positive or negative pole) was determined by whether the QRS complex in the perpendicular lead had a positive or negative deflection. The degree value of the MEA was the pole for the perpendicular lead bearing the determined sign or polarity of the QRS complex of the perpendicular lead. The only exception was lead aVR whose positive pole is located at $-150^{\circ}$ and lead aVL whose positive pole is located at $-30^{\circ}$. In cases where the isoelectric lead was not perfectly isoelectric, the degree value of the MEA was adjusted towards the positive pole (if isoelectric lead showed a more positive deflection) or negative pole (if isoelectric lead showed a more negative deflection) of the isoelectric lead (Bolton 1975).

The electrocardiographic values recorded before and after AW insertion were analyzed using analysis of variance (ANOVA) using SAS (2002). This was done in order to test for differences between the measurements for the various treatments (days) and also between the measurements within a given treatment (day). Differences were taken to be significant at $P<0.05$. 


\section{Results}

None of the dogs were found to be infested with dirofilaria worms when they were examined at necropsy. There were no changes observed in the heart and great vessels except for the presence of the inserted artificial polyvinyl chloride "worms" in the right ventricle and pulmonary artery. There was a significant attenuation of amplitude of Q wave in lead I on days 1-4, 7, 14, 21, 28 and 35; of R wave in lead II on days 21, 28 and 35; and of S wave in lead aVR on days $7,14,21,28$ and 35 following AW insertion $(P<0.05$, Table 1$)$.

Table 1. Amplitude (mV) of Q wave in lead I, R wave in lead II, S wave in lead aVR and duration (s) of QRS in lead aVF recorded from dogs before (baseline, day -1) and after 30 artificial Dirofilaria immitis worms were inserted into the right side of the heart

\begin{tabular}{lcccc}
\hline \multirow{2}{*}{$\begin{array}{l}\text { Day before } \\
\text { or day(s) after } \\
\text { worm insertion }\end{array}$} & \multicolumn{4}{c}{ Wave and lead } \\
\cline { 2 - 5 } & Q1 & Rmplitude & \\
\hline-1 & $0.38 \pm 0.05$ & $2.16 \pm 0.11$ & $1.51 \pm 0.12$ & Duration $^{1}$ \\
1 & $0.21 \pm 0.04^{*}$ & $1.99 \pm 0.18$ & $1.29 \pm 0.11$ & $0.043 \pm 0.001$ \\
2 & $0.19 \pm 0.04^{*}$ & $1.98 \pm 0.17$ & $1.28 \pm 0.14$ & $0.046 \pm 0.002$ \\
3 & $0.23 \pm 0.07^{*}$ & $2.07 \pm 0.21$ & $1.27 \pm 0.13$ & $0.045 \pm 0.002$ \\
4 & $0.21 \pm 0.05^{*}$ & $1.98 \pm 0.19$ & $1.24 \pm 0.11$ & $0.042 \pm 0.001$ \\
7 & $0.16 \pm 0.02^{*}$ & $1.96 \pm 0.19$ & $1.11 \pm 0.09^{*}$ & $0.048 \pm 0.003$ \\
14 & $0.16 \pm 0.04^{*}$ & $1.71 \pm 0.17$ & $1.02 \pm 0.11^{*}$ & $0.051 \pm 0.003^{*}$ \\
21 & $0.16 \pm 0.04^{*}$ & $1.53 \pm 0.10^{*}$ & $1.00 \pm 0.10^{*}$ & $0.041 \pm 0.001$ \\
28 & $0.20 \pm 0.06^{*}$ & $1.50 \pm 0.17^{*}$ & $0.90 \pm 0.11^{*}$ & $0.045 \pm 0.002$ \\
35 & $0.23 \pm 0.05^{*}$ & $1.24 \pm 0.13^{*}$ & $0.85 \pm 0.10^{*}$ & $0.045 \pm 0.003$ \\
\hline
\end{tabular}

${ }^{1}$ Values represent the mean \pm SEM of 12 dogs; *Significantly different from the baseline $(P<0.05)$.

Amplitude of $\mathrm{Q}$ wave $(\mathrm{mV}$; mean \pm SEM) in lead I was significantly reduced from a value of $0.38 \pm 0.05$ recorded prior to AW insertion to a value of $0.21 \pm 0.04,0.19 \pm 0.04$, $0.23 \pm 0.07,0.21 \pm 0.05,0.16 \pm 0.02,0.16 \pm 0.04,0.16 \pm 0.04,0.20 \pm 0.06$ and $0.23 \pm 0.05$ recorded on days $1-4,7,14,21,28$ and 35 , respectively after AW insertion $(P<0.05$, Table $1)$. Amplitude of $\mathrm{R}$ wave $(\mathrm{mV}$; mean \pm SEM) in lead II was significantly reduced from a value of $2.16 \pm 0.11$ recorded prior to $\mathrm{AW}$ insertion to a value of $1.53 \pm 0.10,1.50 \pm 0.17$ and $1.24 \pm 0.13$, recorded on days 21,28 and 35, respectively after AW insertion $(P<0.05$, Table 1). Amplitude of $\mathrm{S}$ wave $(\mathrm{mV}$; mean $\pm \mathrm{SEM})$ in lead aVR was significantly reduced from a value of $1.51 \pm 0.12$ recorded prior to AW insertion to a value of $1.11 \pm 0.09,1.02$ $\pm 0.11,1.00 \pm 0.10,0.90 \pm 0.11$ and $0.85 \pm 0.10$, recorded on days $7,14,21,28$ and 35 respectively, after AW insertion $(P<0.05$, Table 1$)$.

Duration of QRS complex (s; mean \pm SEM) in lead aVF was significantly prolonged from a value of $0.043 \pm 0.001$ recorded prior to AW insertion to a value of $0.051 \pm 0.003$ recorded on day 14 after AW insertion $(P<0.05$, Table 1$)$. Although change in the average mean electrical axis in the frontal plane was not-significant $(P>0.05), 5$ of the $12 \operatorname{dogs}$ $(41.6 \%)$ did show right axis deviation during the period following insertion of AW.

\section{Discussion}

In the present study, there was a significant attenuation of amplitude of $\mathrm{Q}$ wave in lead I on days 1-4, 7, 14, 21, 28 and 35; of R wave in lead II on days 21, 28 and 35; and of S wave in lead aVR on days 7, 14, 21, 28 and 35 following AW insertion. However, all these electrocardiographic changes were within the normal ranges for dogs (Hill 1968). 
Analysis of the QRS complex in lead aVF has been suggested as being useful in following the progression of CHD (Hamlin 1968). The progression of the disease is accompanied by attenuation of the Q wave amplitude and in deepening of S wave (Hamlin 1968). An attenuation of Q wave amplitude has similarly been found in leads I, II, III, CV 6 LL and CV 6 LU (Hamlin 1968; Hill 1971). These QRS changes have been attributed to altered conduction within the dilated or hypertrophied ventricle (Hamlin 1968). Right ventricular dilation or right ventricular hypertrophy may produce patterns nearly identical to those produced by interruption of the right bundle of the conduction system (Hamlin 1968). We have previously shown that insertion of artificial worms into the right heart and pulmonary artery of dogs causes elevation of pulmonary arterial pressures (Ony ango et al. 1992). This increase in pulmonary arterial pressures would initially lead to right ventricular dilation and only much later would it lead to hypertrophy (Rawlings and Lewis 1977; McCall et al. 2008; Seiler et al. 2010). It was recorded that the attenuation of the QRS complex can be due to right ventricular dilation causing altered conduction and also depression of the right ventricular action potentials (Hamlin 1968; Rasmussen and Michelsen 1974).

In the present study, the duration of the QRS complex in lead aVF was significantly longer than the baseline value on day 14 after AW insertion. However, since the duration of the QRS complex was within the normal limits and only significant in one lead, it seems that the duration of the ORS complex is not significant. A significant change in QRS duration will appear in more than one lead.

In the present study, the average mean electrical axis in the frontal plane was within the normal range. However, 5 of the 12 dogs (41.6\%) showed right axis deviation after AW insertion. Clockwise shift of the mean electrical axis has been reported to occur during pulmonary obstruction (Love et al. 1938). This has been attributed to simple anatomical rotation of the heart which may occur during right heart strain (Ras mus sen and Michels en 1974). Insertion of artificial worms in the pulmonary tree of a dog elevates pulmonary and right ventricular pressures (Onyango et al. 1992). This elevation of pressures could have been the cause of the right heart strain. The increased pressure could also result in right ventricular dilation reported in canine heartworm disease (Lombard and Ackerman 1977; Rawlings and Lew is 1977). Calvert et al. (2005) have also reported change in the mean electrical axis in dogs with heartworm disease.

Based on our results, early canine heartworm disease can be diagnosed by the comparison of the QRS complex of the baseline and subsequent standard electrocardiograms. In early $\mathrm{CHD}$, an attenuation of amplitude of Q wave in lead I, R wave in lead II and S wave in lead aVR is recorded. Some dogs with early CHD can also show right axis deviation. A more comprehensive study to monitor not only the ECG but also the timeline for the morphological progression of the artificial worm infestation is needed and can bring more information on canine heartworm infestation.

\section{Acknowledgements}

I wish to thank the Kenya Medical Research Institute for allowing the use of their fluoroscopic facility for this study.

\section{References}

Atkins CE 2003: Comparison of results of three commercial heartworm antigen test kits in dogs with low heartworm burdens. J Am Vet Med Assoc 222: 121-1223

Atwell RB, Buoro IBJ, Sutton RH 1985: Experimental production of lesions in canine pulmonary arteries similar to those produced by Dirofilaria immitis infection. Vet Rec 116: 539-541

Bolton GR 1975: Handbook of canine electrocardiography. W.B. Saunders Company, Philadelphia, 370 p.

Calvert CA, Losonsky JM, Brown J, Lewis RE 2005: Comparisons of radiographic and electrocardiographic abnormalities in canine heartworm disease. Vet Radiol Ultrasound 27: 2-7

Ettinger SJ, Suter PF 1970: Canine cardiology. W.B. Saunders Company, Philadelphia, 616 p.

Hamlin RL 1968: Electrocardiographic detection of ventricular enlargement in the dog. J Am Vet Med Assoc 153: $1461-1469$ 
Hill JD 1968: The electrocardiogram in dogs with standardized body and limb positions. J Electrocardiol 1: $175-182$

Hill JD 1971: Electrocardiographic diagnosis of right ventricular enlargement in dogs. J Electrocardiol 4: $347-357$

Knight DH 1983: Heartworm Disease. In: Ettinger SJ (Ed.): Textbook of Veterinary Internal Medicine: Diseases of the Dog and Cat, Vol 1, 2nd edn. W. B. Saunders Company, Philadelphia, pp. 1097-1124

Lombard CW, Ackerman N 1977: Right heart enlargement in heartworm-infected dogs. Vet Radiol Ultrasound 25: $210-217$

Love WS JR, Brugler GW, Winslow N 1938: Electrocardiographic studies in clinical and experimental pulmonary embolization. Ann Intern Med 11: 2109-2123

McCall JW, Genchi C, Kramer LH, Guerrero J, Venco L 2008: Heartworm disease in Animals and Humans. Adv Parasitol 66: 193-285

Onyango EM, Buoro IBJ, Kanui TI 1992: Induction of pulmonary hypertension in the dog using polyvinyl chloride threads. Israel J Vet Med 49: 31-35

Otto GF, Jackson RF 1975: Heartworm disease. In: Ettinger SJ (Ed.) Textbook of Veterinary Internal Medicine: Diseases of the dog and cat, Vol 2. W. B. Saunders Company, Philadelphia, pp. 1014-1038

Rasmussen K, Michelsen K 1974: The effect of acute pulmonary artery obstruction on the dog electrocardiogram. Am Heart J 87: 209-216

Rawlings CA, Lewis RE 1977: Right ventricular enlargement in heartworm disease. Am J Vet Res 38: $1801-1805$

Rishniw M, Barr SC, Simpson KW, Frongillo MF, Franz M, Dominguez Alpizar JL 2006: Discrimination between six species of canine microfilariae by a single polymerase chain reaction. Vet Parasitol 135: 303-314

SAS 2002: Statistical Analysis System Proprietary Software. Release 8.1. SAS Institute Inc., Cary, NC

Seiler GS, Nolan TJ, Withnall E, Reynolds C, Lok JB, Sleeper MM 2010: Computed tomographic changes associated with the prepatent and early patent phase of dirofilariasis in an experimentally infected dog. Vet Radiol Ultrasound 51: 136-140

Strickland HH 2008: Canine and feline dirofilariasis: lifecycle, pathophysiology, and diagnosis. Compend Contin Educ Vet 30: 133-140 